\title{
Les enquêtes « Enveff » sur les violences envers les femmes dans la France hexagonale et ultramarine
}

The "Enveff" studies on violence against women in France and its overseas territories

\section{Elizabeth Brown}

\section{(2) OpenEdition}

\section{Journals}

Édition électronique

URL : http://journals.openedition.org/plc/860

DOI : $10.4000 /$ plc. 860

ISSN : 2117-5209

Éditeur

L'Harmattan

Édition imprimée

Date de publication : 1 janvier 2012

Pagination : 43-59

ISBN : 978-2-296-55-856-4

ISSN : $1279-8657$

\section{Référence électronique}

Elizabeth Brown, «Les enquêtes « Enveff » sur les violences envers les femmes dans la France hexagonale et ultramarine », Pouvoirs dans la Caraïbe [En ligne], 17 | 2012, mis en ligne le 26 janvier 2012, consulté le 19 avril 2019. URL : http://journals.openedition.org/plc/860 ; DOI : 10.4000/plc.860 


\title{
LES ENQUÊTES «ENVEFF » SUR LES VIOLENCES ENVERS LES FEMMES DANS LA FRANCE HEXAGONALE ET ULTRAMARINE
}

\author{
Elizabeth BROWN \\ Directrice du Centre de recherche de \\ l'Institut de démographie \\ Université Paris 1 Panthéon-Sorbonne
}

\section{I. - MISE EN PLACE}

En France, le rapport préparatoire établi pour la conférence de Beijing $^{1}$ avait retenu le thème des violences parmi les axes prioritaires de la contribution française et demandait la production de «statistiques précises concernant les violences faites aux femmes ». En effet, les statistiques criminelles publiées par les ministères de l'Intérieur, de la Défense ou de la Justice portaient davantage sur les auteurs que sur les victimes. Les études réalisées par divers observatoires et centres de recherche restaient ponctuelles - dans l'espace ou dans le temps - et, dans les enquêtes nationales conduites par des institutions comme l'Insee ou l'Inserm ${ }^{2}$, les modules consacrés au thème des violences étaient trop courts pour cerner vraiment le phénomène et décrire en détail le processus de son développement. Quant aux rapports des associations de lutte contre les violences $^{3}$, ils décrivaient bien la situation des victimes, mais ne permettaient pas d'évaluer l'ampleur de ces violences dans l'ensemble de la population.

C'est ainsi qu'en 1996, à la suite de la Conférence de Beijing, le Service des Droits des Femmes du Ministère de l'Emploi et de la Santé a demandé à Michel Bozon (Ined) de mettre en place une enquête nationale sur le sujet - avec pour objectif la production de statistiques fiables, portant sur l'ensemble de la population féminine et permettant de mesurer les divers types de violences interpersonnelles (verbales, psychologiques, physiques et sexuelles) subies par les femmes. L'année suivante, une équipe

\footnotetext{
${ }^{1}$ Claire Aubin \& Hélène Gisserot. Les femmes en France 1985-1995. Paris, la Documentation française, 1994.

${ }^{2}$ Enquêtes permanentes sur les conditions de vie des ménages (EPCV), depuis 1996 ; enquête sur les comportements sexuels en France (ACSF), 1992 ; enquête sur les comportements sexuels des jeunes en France (ACSJ), 1994.

${ }^{3}$ Fédération nationale solidarité femmes, Collectif féministe contre le viol, Association européenne contre les violences faites aux femmes au travail.
} 
pluridisciplinaire de chercheurs appartenant à plusieurs universités et organismes de recherche, coordonnée par l'Institut de Démographie de l'Université Paris 1 (Idup) et dirigée par Maryse Jaspard, commençait les travaux de l'Enquête nationale sur les violences envers les femmes en France, dite Enveff. A la différence des statistiques administratives ou associatives, issues de l'activité des institutions (police, justice, services d'écoute ou d'hébergement, etc.), il s'agissait de réaliser une enquête dite de victimation, reposant sur l'auto-déclaration des violences (éventuellement) subies par des personnes interrogées à la suite d'un processus de sélection aléatoire et non sur l'enregistrement administratif, policier ou pénal de faits de violence déclarés par les victimes ou constatés par la police ou la gendarmerie. La collecte des données a été menée dans l'hexagone de mars à juillet 2000, auprès d'un échantillon représentatif de 6970 femmes âgées de vingt à cinquante-neuf ans et résidant hors institution, sélectionnées aléatoirement à partir de listings téléphoniques et interrogées anonymement. Elle a été effectuée par un institut de sondage utilisant un système de collecte assistée par téléphone et informatique (dit Cati).

Par la suite, des enquêtes similaires ont été menées dans la plupart des départements, territoires et pays d'Outre-mer. Pour les départements d'Outre-mer, l'initiative en revient aux acteurs de terrain et aux chercheurs qui sont parvenus à sensibiliser les Secrétariats d'Etat au droit des femmes et à l'Outre-mer. Ce dernier a été le principal financeur de la première étape dans les départements français d'Amérique (DFA), sous la responsabilité de Myriam Cottias ${ }^{4}$, et à La Réunion, sous la responsabilité d'Isabelle Widmer : quatre enquêtes pilotes ont ainsi été effectuées en 2001 sur des échantillons de 150 à 200 femmes de chaque département. Mais, par suite du désengagement des deux Secrétariats d'Etat, seule La Réunion a pu réaliser, en 2002, à l'aide de financements essentiellement locaux, l'enquête en vraie grandeur sur un échantillon représentatif ${ }^{5}$. En Polynésie, le recueil des données a été impulsé en 2002 par des acteurs de terrain en liaison avec le

\footnotetext{
${ }^{4}$ L'enquête pilote pour les départements français d'Amérique a été effectuée dans le cadre du Centre de recherche sur les pouvoirs locaux dans la Caraïbe (Université des Antilles et de la Guyane) par Myriam Cottias, responsable scientifique, Nadine Lefaucheur et Stéphanie Mulot. Plusieurs autres personnes ont été associées à différentes phases de cette enquête (en particulier Arlette Gautier, Maryse Jaspard, Elizabeth Brown, Myriane Joly, Mylenn Zobda, Pascale Benoît, Véronique Hélénon, Fanny Thomas, Patrice Lefaucheur et Damien Valdant).

${ }^{5}$ Isabelle Widmer. Enquête nationale sur les violences envers les femmes en France à l'île de La Réunion, Enveff-Réunion. Rapport final de l'enquête quantitative. INED, 2003 ;

Dolorès Pourette. Enquête nationale sur les violences envers les femmes en France à l'île de La Réunion, Enveff-Réunion. Rapport final de l'enquête qualitative. INED, 2004 ;

Dolorès Pourette \& Isabelle Widmer. «La violence sur la place publique et dans le couple». Dossier «Femmes et hommes à parité ? ", Revue de l'INSEE-Réunion: Economie de La Réunion, $\mathrm{n}^{\circ} 122,4^{\circ}$ trimestre 2004, p. 20-22;

Isabelle Widmer \& Dolorès Pourette. Les violences envers les femmes à l'île de La Réunion. Poids des chiffres et paroles de victimes. Publications de l'Université de Provence, 2009.
} 
Ministère de la santé du territoire ${ }^{6}$. En Nouvelle-Calédonie, des anthropologues ont trouvé des financements dans le cadre de l'Inserm et réalisé, en 2003, l'enquête "Santé, conditions de vie et de sécurité des femmes calédoniennes $»^{7}$. En Polynésie et à La Réunion, des entretiens semi-directifs ont également été réalisés par des anthropologues ${ }^{8}$.

\section{II. - PRINCIPES ET PROBLÉMATIQUES}

L'enquête nationale sur les violences faites aux femmes en France (Enveff) a été présentée aux personnes sollicitées comme une enquête sur la santé et la sécurité et le parti a été pris de ne pas employer les mots « violence » et « violent », mais de nommer ou décrire précisément les actes violents (insultes, menaces et agressions verbales, chantage ou pressions psychologiques, agressions physiques, viol et pratiques sexuelles imposées) dont les femmes avaient pu être victimes dans l'espace public, au travail, dans le couple, dans la famille ou de la part de proches.

Les questions posées aux femmes sur les actes violents subis, soit au cours des douze derniers mois, soit, pour les plus graves, au cours de leur vie entière, n'intervenaient qu'après le recueil d'informations portant sur leurs caractéristiques familiales, économiques, sociales et résidentielles, sur leur parcours biographique, sur leur état et pratiques de santé et sur l'entente conjugale au quotidien (répartition des tâches ménagères et des soins aux

\footnotetext{
${ }^{6}$ Maryse Jaspard, Elizabeth Brown \& Claudine Pirus. Enquête sur les violences envers les femmes en Polynésie française. Rapport final. IDUP, 2003 ;

Maryse Jaspard, Elizabeth Brown \& Dolorès Pourette. «Les violences envers les femmes dans le cadre du couple en Polynésie française ». Espaces, Populations, Sociétés, n 2, 2004 ; Dolorès Pourette. Les violences envers les femmes en Polynésie française. Etude socioanthropologique. Rapport d'analyse. Ministère de la Santé de Polynésie française, direction de la santé, 2002.

Pour une comparaison des données recueillies en métropole, à La Réunion, en NouvelleCalédonie et en Polynésie française, cf. Elizabeth Brown \& Isabelle Widmer. «Les violences envers les femmes en France : Continuités et différences au-delà des mers », in Violences envers les femmes, trois pas en avant, deux pas en arrière. Paris, l'Harmattan, 2007.

7 Christine Salomon, Christine Hamelin et al. Premiers résultats de l'enquête santé, conditions de vie et de sécurité des femmes calédoniennes. Rapport INSERM U88, INSERM, 2004 ;

Christine Hamelin \& Christine Salomon. «Parenté et violences faites aux femmes en Nouvelle-Calédonie. Un éclairage sur l'ethnicité différenciée des violences subies au sein de la famille ». Espaces, Populations, Sociétés, 2004, n 1, p. 307-323;

Christine Hamelin, Christine Salomon, Alice Gueguen, France Lert, Diane Cyr. «Abus sexuels précoces et santé reproductive des femmes en Nouvelle-Calédonie 2002-2003», B.E.H., 2005, n ${ }^{\circ} 9-10$, p. 33-35.

${ }^{8}$ Dolorès Pourette. «Paroles et sexualité dans le couple à La Réunion et en Polynésie française », in M. Jaspard \& N. Chetcutti (dir.). Violences envers les femmes, trois pas en avant, deux pas en arrière. Paris, 1'Harmattan, 2007. p. 241-256.
} 
enfants, disputes, etc.). Ces informations étaient destinées à être mises en relation avec les faits de violence subis: s'il apparaissait important de pouvoir quantifier ceux-ci, il le semblait en effet encore plus de tenter de mieux appréhender leur origine et leur contexte d'apparition.

Les concepteurs du questionnaire ont considéré, « conformément aux acquis théoriques sur le sujet ${ }^{9}$, que les violences au sein des couples formaient un continuum incluant les agressions verbales, psychologiques, physiques et sexuelles et que "par-delà les actes violents caractérisés brutalités physiques et sexuelles -, l'accumulation de faits, de gestes, de paroles en apparence sans gravité crée des situations pouvant porter gravement atteinte aux personnes ». Différentes questions portaient ainsi sur les atteintes à la dignité et à la personnalité, les critiques et dévalorisations systématiques, le contrôle de l'apparence physique, des activités et relations, le refus de communication ou celui de l'accès aux revenus du ménage, et le chantage à propos des relations avec les enfants. Des indicateurs combinant la nature, la multiplicité et la fréquence des faits déclarés ont été construits pour en évaluer la gravité.

Comme la plupart des enquêtes nationales sur la violence sexuée ${ }^{10}$ entreprises dans les années $1990^{11}$, l'enquête Enveff s'inscrit dans le cadre des problématiques de genre et de «domination masculine». Au contraire des études pionnières, la violence n'y est pas considérée comme une modalité, même extrême, de gestion d'un conflit conjugal : "Contrairement au conflit, mode relationnel interactif susceptible d'entraîner du changement, la violence - signalant d'ailleurs l'incapacité à communiquer - est perpétrée de façon univoque et destructrice. (...) le mécanisme de violence se met en place dès que le vainqueur de l'altercation est toujours le même $»^{12}$.

L'enquête Enveff se distingue cependant de la plupart des autres enquêtes nationales en ne focalisant pas sur la violence conjugale, mais en envisageant tous les espaces de vie où les femmes peuvent subir des violences. Par ailleurs, les chercheurs n'ont pas considéré que les violences subies par les femmes étaient toujours le fait d'hommes (pour toute réponse

\footnotetext{
${ }^{9}$ Maryse Jaspard \& al. «Réponse à Marcela Iacub, Hervé Le Bras : Violences vécues, fantasmes et simulacres... ». Les Temps modernes, mai-juin-juillet 2003, p. 184-195.

${ }^{10} \mathrm{Y}$ compris les enquêtes sur la santé des femmes, qui comportent souvent un module sur les actes de violence subis par les femmes.

${ }^{11}$ Cf. Lucienne Gilliot, Jacqueline De Puy, Véronique Ducret. Domination et violence envers la femme dans le couple. Lausanne, Payot, 1997 ;

Markku Heiskanen \& Minna Piispa. Faith, Hope, Battering. A Survey of Men's Violence in Finland. Helsinki, Statistics Finland, 1998.

${ }_{12}$ Maryse Jaspard et al. Les violences envers les femmes en France. Une enquête nationale. Paris, la Documentation française, 2002, p. 18.
} 
positive, il était demandé de préciser le sexe de l'auteur de l'acte violent), ni que les femmes étaient seulement victimes et non auteurs de violences ${ }^{13}$.

Cette prise en compte, certes limitée, de violences commises par les femmes n'a pas empêché que cette enquête de victimation soit, dès la publication des premiers résultats ${ }^{14}$, la cible de vives critiques l'accusant de victimiser les femmes, la regardant comme «l'exemple paradigmatique » d'un «féminisme victimiste» qui fournirait « une nouvelle version de la femme-mère, afin d'en faire le garant de la reproduction et de la famille ${ }^{15}$, et condamnant une «démarche victimiste » qui amènerait le féminisme français à faire « fausse route ${ }^{16} »$.

D'autres critiques (en particulier du Conseil National de l'Information Statistique) ont parfois reproché à l'équipe de recherche de ne s'être intéressée qu'aux violences subies par les femmes. Si les chercheurs ont reconnu que prendre en compte la population masculine dans l'enquête ou que mener une enquête de même type sur cette population aurait été souhaitable, et que la violence ne s'exerce pas de façon univoque des hommes vers les femmes, ils ont néanmoins estimé que «le peu d'études (alors) disponibles sur les hommes ne permettaient pas d'élaborer la problématique d'une enquête quantitative sur le sujet ${ }^{17}$. Ils estimaient également qu'il n'était pas anormal de donner la priorité à la mesure des violences subies par les femmes, la violence sexuée étant asymétrique, même lorsqu' on pouvait la regarder comme réciproque : «la première constatation sociologique est que ce sont les femmes qui sont très majoritairement victimes des violences conjugales et des violences sexuelles. $\mathrm{Si}$ violence et non-violence ne permettent pas de tracer une ligne de partage entre les sexes, les rapports de domination engendrent des actes de violence et la violence masculine peut être analysée comme un mécanisme

\footnotetext{
${ }^{13}$ Il était ainsi demandé à l'enquêtée s'il lui était arrivé, au cours des douze derniers mois, «a) de crier très fort ou de dire des insultes quand (elle était) en colère ; b) de gifler, donner une fessée ou une tape à un enfant ; c) de gifler ou de frapper une personne adulte ». Il lui était également demandé, lorsqu'elle avait une relation de couple, si elle s'était disputée avec son conjoint ou ami au cours des douze derniers mois, si oui, à quel propos, et si, lors de disputes, seulement elle, seulement son conjoint, ou les deux en étaient venus aux mains, avaient proféré des propos blessants, lancé ou cassé des objets.

${ }^{14}$ Maryse Jaspard \& al. « Nommer et compter les violences envers les femmes : une première enquête nationale en France ». Population et sociétés, nº 364, 2001.

${ }^{15}$ Marcela Iacub, Hervé Le Bras. «Homo mulieri lupus? A propos d'une enquête sur les violences envers les femmes ». Les Temps modernes, février-mars-avril, 2003, p. 112-134.

${ }^{16}$ Elizabeth Badinter. Fausse route. Paris, Odile Jacob, 2003. Elizabeth Badinter, qui critique violemment l'enquête Enveff, définit le «victimisme » comme «un néologisme qui désigne l'attitude consistant à se définir en priorité comme une victime » (p. 18).

${ }^{17}$ Maryse Jaspard et al. « Réponse à Marcela Iacub, Hervé Le Bras... », 2003. op. cit.
} 
fondamental de contrôle des femmes (Hanmer, $1977^{18}$ ). Bien que les femmes puissent elles-mêmes être auteurs de violences lorsqu'elles se trouvent en situation de pouvoir, les violences qu'elles subissent expriment leur position de dominées, ce qui n'exclut pas de leur part des réactions, y compris violentes, à cette situation ${ }^{19}$.

\section{III. - LES ENQUÊTES ENVEFF OUTRE-MER}

Les enquêtes sur les violences envers les femmes réalisées dans les DOM-TOM dans le sillage de l'enquête Enveff, n'ont apporté à ce modèle que des modifications limitées: adaptation du vocabulaire utilisé et des questions portant sur le contexte aux réalités locales, réalisation de l'enquête en face à face lorsque la couverture téléphonique ne permettait pas l'enquête par téléphone, constitution de l'échantillon à partir de la méthode des quotas ou par tirage au sort sur les listes électorales et non sur des listings téléphoniques.

Les équipes pluridisciplinaires de chercheurs ${ }^{20}$, appartenant aux institutions publiques de recherche, ont en effet eu le souci de travailler en étroite collaboration afin de produire des résultats comparables. L'objectif commun était de mesurer, pour l'ensemble de la population de chaque territoire, les divers types de violences interpersonnelles (verbales, psychologiques, physiques et sexuelles) afin de mieux cerner le phénomène social des violences envers les femmes. Si la formulation des questions a été adaptée aux contextes sociaux et culturels, la structure du questionnaire est demeurée identique.

La collecte des données a été menée auprès d'échantillons représentatifs de femmes âgées de 18 ou 20 ans à 54 ou 59 ans et résidant dans des ménages ordinaires (voir tableau 1). Dans l'Hexagone et à La

\footnotetext{
${ }^{18}$ Jalna Hanmer. «Violence et contrôle social des femmes », Questions féministes, n 1, 1977, p. 69-88.

${ }^{19}$ Maryse Jaspard et al. Les violences envers les femmes en France. Une enquête nationale, 2002. op. cit. p. 18.

${ }^{20}$ L'équipe Enveff Hexagone était composée de Maryse Jaspard, responsable de l'enquête, Elizabeth Brown, Stéphanie Condon, Jean-Marie Firdion, Dominique Fougeyrollas-Schwebel, Annik Houël, Brigitte Lhomond, Florence Maillochon, Marie-Josèphe Saurel-Cubizolles, Marie-Ange Schiltz.

Isabelle Widmer, responsable scientifique de l'enquête à La Réunion, a travaillé en particulier avec Christine Catteau et Dolorès Pourette.

Pour la Polynésie française, Maryse Jaspard, responsable de l'enquête, était associée à Elizabeth Brown et Claudine Pirus.

Enfin, l'équipe de Nouvelle-Calédonie rassemblait Christine Salomon, Christine Hamelin, Pâquerette Goldberg, Rémi Sitta, Diane Cyr, Alice Gueguen, France Lert, Marcel Goldberg et Jean-Pierre Nakache.
} 
Réunion (ainsi que dans les départements français d'Amérique (DFA) pour l'enquête pilote), elle a été effectuée par téléphone, selon la méthode Cati. En Polynésie et en Nouvelle-Calédonie, l'équipement téléphonique des ménages s'avérant trop inégal, la procédure en face à face a été retenue.

Tableau 1 : Caractéristiques des quatre enquêtes sur les violences envers les femmes réalisées en 2000-2003 en France hexagonale et ultramarine

\begin{tabular}{|c|c|c|c|c|c|c|c|c|}
\hline \multirow[t]{2}{*}{ Localisation } & \multicolumn{2}{|c|}{$\begin{array}{c}\text { Femmes } \\
\text { interrogées }\end{array}$} & \multirow[t]{2}{*}{ Méthode } & \multicolumn{5}{|c|}{ Modules concernant les violences } \\
\hline & 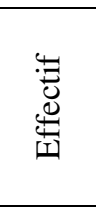 & 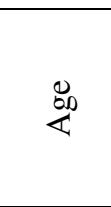 & & 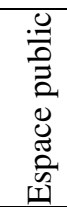 & 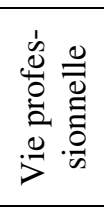 & 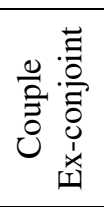 & 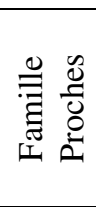 & $\begin{array}{l}\stackrel{0}{0} \\
\stackrel{0}{0} \\
\stackrel{0}{>} \\
\stackrel{0}{>}\end{array}$ \\
\hline Hexagone & 6970 & $20-59$ & Cati & oui & oui & oui & oui & oui \\
\hline Réunion & 1213 & $20-59$ & Cati & oui & oui & oui & & oui \\
\hline \begin{tabular}{|l|} 
Polynésie \\
\end{tabular} & 1001 & $18-59$ & Face-à-face & oui & $*$ & oui & & oui \\
\hline $\begin{array}{l}\text { Nouvelle- } \\
\text { Calédonie }\end{array}$ & 1012 & $18-54$ & Face-à-face & oui & $*$ & oui & oui & oui \\
\hline
\end{tabular}

\section{IV. - LES PRINCIPAUX RÉSULTATS DES ENQUÊTES ENVEFF DANS L'HEXAGONE, À LA RÉUNION ET DANS LE PACIFIQUE}

Dans tous les territoires, la relation conjugale ou amoureuse est le principal théâtre des violences que les femmes ont déclaré avoir subies au cours de l'année écoulée. Viennent ensuite les espaces publics. La vie professionnelle ou estudiantine apparaît moins perturbée, du moins dans l'hexagone et à La Réunion, où elle a fait l'objet d'une interrogation spécifique. Ce sont les relations avec la famille et les amis qui semblent les plus exemptes d'agressions à l'âge adulte, peut-être parce que ce sont celles auxquelles, majeures, les enquêtées ont pu le moins difficilement cesser de s'exposer lorsque ces relations étaient psychologiquement, physiquement ou sexuellement violentes. 


\section{A. - Les violences au cours des douze derniers mois}

\section{1. - Violences}

La relation conjugale envisagée dans les enquêtes Enveff est celle de deux personnes ${ }^{21}$ mariées ou vivant maritalement, ou de deux personnes ayant des relations amoureuses ou sexuelles suivies sans partager un même logement.

Dans l'hexagone et à La Réunion, une femme sur quatre engagées dans une telle relation au moment de l'enquête ${ }^{22}$ est apparue soumise à des pressions psychologiques qui, dans un cas sur trois, pouvaient être qualifiées de harcèlement, du fait de leur répétition; dans les deux territoires de l'océan Pacifique, les taux étaient nettement plus élevés, en particulier pour ce qui relève du harcèlement (tableau 2). Moins fréquemment déclarées, les insultes et injures proférées par le conjoint ou ami au cours de l'année écoulée l'ont cependant été par environ $4 \%$ des femmes dans l'hexagone et à La Réunion, et par $21 \%$ des Polynésiennes et $28 \%$ des NéoCalédoniennes $^{23}$. Ces dernières se démarquent encore des habitantes de l'hexagone et de La Réunion ${ }^{24}$ par des taux de violences physiques et sexuelles déclarées sept fois plus élevés : 17 à $19 \%$ des premières pour les agressions physiques versus 2 à $3 \%$ des secondes, $7 \%$ des premières pour les agressions sexuelles versus $1 \%$ pour les secondes. Trois Polynésiennes et sept Néo-Calédoniennes sur cent ont également déclaré que des actes de violence à la fois physique et psychologique tels que le saccage ou l'incendie délibéré de leur maison, de leur voiture ou de leurs affaires personnelles avaient été perpétrés au moins une fois au cours des douze derniers mois par leur conjoint ou ami.

\footnotetext{
${ }^{21}$ Il peut s'agir de deux femmes, mais les couples homosexuels observés sont très peu nombreux ( $0,1 \%$ des couples, cohabitant ou non, déclarés dans l'échantillon hexagonal).

${ }^{22}$ Nombre de celles qui avaient eu un partenaire dans les 12 derniers mois mais en étaient séparées au moment de l'enquête - souvent jeunes et peu nombreuses parmi les femmes enquêtées (de $2 \%$ dans l'hexagone à $6 \%$ en Nouvelle-Calédonie) - avaient, à l'évidence, vécu des situations très conflictuelles, souvent accompagnées de violences, pendant la période de séparation. Elles ont d'ailleurs déclaré de deux à six fois plus d'atteintes conjugales de toutes sortes que les autres enquêtées.

${ }^{23}$ Natives ou immigrées.

${ }^{24}$ Le mode de sélection de l'échantillon et d'interrogation choisi (entretiens téléphoniques après tirage au sort des numéros de téléphone dans l'hexagone et à La Réunion ; entretiens en face-à-face et sélection selon la méthode des quotas en Polynésie ; entretiens en face-à-face, avec une partie auto-administrée, après tirage au sort à partir des listes électorales en Nouvelle-Calédonie) n'est peut-être pas sans impact sur les niveaux de violences enregistrés : les prévalences sont systématiquement plus élevées dans les deux pays d'Océanie, où l'enquête s'est faite en face-à-face, que dans l'hexagone et à La Réunion, où les taux enregistrés par une collecte téléphonique semblent plutôt des seuils minima.
} 
Tableau 2 : Proportion de femmes victimes de violences conjugales dans les douze derniers mois, selon le territoire et le type de violence conjugale

\begin{tabular}{|c|c|c|c|c|}
\hline Violences conjugales & $\begin{array}{c}\text { Métropole } \\
N=5793\end{array}$ & $\begin{array}{c}\text { La Réunion } \\
N=1013\end{array}$ & $\begin{array}{c}\text { Polynésie } \\
N=770\end{array}$ & $\begin{array}{c}\text { Nlle-Calédonie } \\
N=792\end{array}$ \\
\hline Agressions verbales & 4,0 & 4,2 & 21,0 & 27,8 \\
\hline Pressions psychologiques & 23,5 & 27,4 & 36,0 & 41,3 \\
\hline Dont harcèlement & 7,3 & 8,8 & 24,0 & 24,0 \\
\hline Agressions physiques & 2,3 & 2,6 & 17,0 & 19,0 \\
\hline Agressions sexuelles & 0,8 & 1,1 & 7,0 & 7,2 \\
\hline \multicolumn{5}{|c|}{$\begin{array}{l}\text { Champs : ensemble des femmes en couple au moment de l'enquête } \\
\text { Agressions verbales : au moins une fois dans l'année } \\
\text { Pressions psychologiques : au moins trois types de pressions subies «quelquefois » } \\
\text { Harcèlement psychologique : plus de trois types de pressions subies dont au moins un «souvent» } \\
\text { Agressions physiques : gifles, coups, bousculades, menaces avec arme, tentative de meurtre, } \\
\text { séquestration ou mise à la porte } \\
\text { Agressions sexuelles : gestes sexuels imposés, rapports sexuels imposés par la force }\end{array}$} \\
\hline
\end{tabular}

Partout, les femmes jeunes, celles qui ne sont pas mariées et surtout celles qui n'habitent pas avec leur conjoint - trois caractéristiques qui vont souvent de pair - ont été les plus nombreuses à déclarer avoir subi des faits de violence conjugale. Ainsi, la fréquence des pressions psychologiques décroît sensiblement avec l'âge, en particulier dans les territoires ultramarins où une femme sur deux est concernée avant 25 ans, puis seulement une sur quatre (ou une sur six en Polynésie) après 45 ans ; dans l'hexagone, moins élevée parmi les plus jeunes, cette fréquence régresse aussi moins nettement avec l'âge (de $34 \%$ à $21 \%$ ).

Si les unions les plus anciennement constituées apparaissent comme les moins empreintes de violences, c'est sans doute qu'au fil du temps, la cohabitation puis le mariage ont souvent scellé les relations conjugales harmonieuses - ou simplement acceptables - tandis que les relations marquées par la violence étaient plus susceptibles de prendre fin: la mésentente, l'absence d'amour et l'infidélité sont fortement corrélées aux cumuls de violences conjugales dans les quatre territoires.

Outre-mer, la fréquente proximité résidentielle, voire la cohabitation, avec la famille d'un des conjoints exacerbe les violences conjugales en accroissant le contrôle exercé sur les femmes, doublé d'un chantage à propos des enfants : souvent chargées d'une part importante des tâches domestiques et étroitement surveillées, toujours critiquées, les jeunes mères ne peuvent quitter leur conjoint sous peine d'être séparées de leurs enfants. Par ailleurs, la consommation abusive d'alcool, du conjoint mais aussi de la femme, y apparaît encore plus liée à l'accroissement des agressions que dans l'hexagone. 
L'influence des caractéristiques sociales du couple sur le niveau de violence conjugale varie également selon les territoires. Si une situation précaire, souvent consécutive au chômage ou à l'éviction de l'emploi de l'un ou l'autre des partenaires accroît toujours les risques, une formation et une situation professionnelle élevées semblent protéger les femmes résidant dans les îles alors que tel n'est pas le cas en France continentale.

L'hypothèse de la reproduction de la violence, selon laquelle les victimes et les auteurs répètent les situations violentes vécues dans leur enfance, semble se vérifier dans les quatre territoires. Parmi les difficultés éprouvées dans l'enfance ou l'adolescence, ont été principalement cités les sévices, les placements par l'assistance publique ou mesures d'assistance éducative, les conflits entre l'enquêtée et ses parents, les problèmes d'alcoolisme et de drogue et le fait d'avoir été témoin de violences entre les parents $^{25}$. Les taux de violences physiques et sexuelles subies dans l'enfance et l'adolescence sont élevés dans les quatre territoires, mais particulièrement en Polynésie et en Nouvelle-Calédonie. Selon la gravité de ces situations, les taux de violence conjugale croissent, jusqu'à tripler parfois, dans tous les territoires et les indices de cumul de violences augmentent encore davantage. Beaucoup de femmes ayant subi des difficultés dans l'enfance ne connaissent heureusement pas de violence conjugale à l'âge adulte, mais les phénomènes de répétition apparaissent d'autant plus massifs que les expériences douloureuses ont été plus fréquentes aux jeunes âges. Ces phénomènes affectent moins les femmes habitant l'hexagone que celles qui vivent dans les îles du Pacifique : parmi les femmes qui ont été victimes de sévices dans leur enfance, près des trois quarts $(72 \%)$ des habitantes de l'hexagone n'ont pas déclaré avoir été victimes de violences conjugales à l'âge adulte, contre la moitié de celles qui vivent en Nouvelle-Calédonie et en Polynésie. La reproduction sociale de la violence semble renforcée lorsqu'il y a cumul de situations de précarité : précarité économique, chômage ou perte d'emploi du conjoint, alcoolisme, etc.

\section{2. - Les violences dans l'espace public}

Dans la rue, les transports en commun, les lieux de loisir, etc., abondent les agressions verbales et atteintes sexuelles (gestes grivois, attouchements). Si les agressions physiques et sexuelles y sont moins fréquentes, les Polynésiennes et Calédoniennes courent, là encore, plus de risques que les métropolitaines et les Réunionnaises (tableau 3). Partout, les auteurs des agressions rapportées sont majoritairement des hommes; souvent connus de leurs victimes, ils les ont agressées dans des lieux

\footnotetext{
${ }^{25}$ Notons, en revanche, que le fait d'avoir vécu la séparation ou le divorce des parents semble de peu d'influence sur le risque de subir des violences conjugales à l'âge adulte.
} 
familiers, de jour comme de nuit. Les femmes jeunes, celles qui n'ont pas de conjoint ou ne vivent pas avec lui, mais aussi les étudiantes, les salariées et les habitantes des grandes villes sont les plus atteintes: sortant davantage, souvent seules, utilisant les transports publics, elles évoluent dans un espace traditionnellement investi par les hommes et qui reste « masculin ${ }^{26}$.

Tableau 3 : Proportion de femmes ayant subi des agressions dans les espaces publics dans les douze derniers mois, selon le territoire et le type de violence

\begin{tabular}{|c|c|c|c|c|}
\hline Espace public* & $\begin{array}{c}\text { Métropole } \\
N=6970\end{array}$ & $\begin{array}{c}\text { La Réunion } \\
N=1213\end{array}$ & $\begin{array}{c}\text { Polynésie } \\
N=1001\end{array}$ & $\begin{array}{c}\text { Nlle-Calédonie } \\
N=1012\end{array}$ \\
\hline Agressions verbales & 13,2 & 11,0 & 15,0 & 17,0 \\
\hline Atteintes sexuelles & 8,3 & 11,7 & 6,0 & 11,0 \\
\hline Agressions physiques & 1,7 & 2,8 & 5,0 & 4,0 \\
\hline Agressions sexuelles & 0,1 & 0,2 & 1,0 & 2,0 \\
\hline \multicolumn{5}{|c|}{$\begin{array}{l}\text { Champs : ensemble des femmes enquêtées dans chaque territoire } \\
* \text { : pour la Polynésie et la Nouvelle-Calédonie, les lieux de travail sont inclus dans l'espace public } \\
\text { Atteintes sexuelles : suivie avec insistance, exposée à l'exhibitionnisme, pelotée } \\
\text { Agressions physiques : vol avec violence, giflée, frappée, menacée avec une arme } \\
\text { Agressions sexuelles : tentative de viol ou viol }\end{array}$} \\
\hline
\end{tabular}

\section{3. - Les violences au travail}

Mises à part les brutalités et menaces physiques qui apparaissent beaucoup plus fréquentes à La Réunion, les différents taux d'agressions au travail (tableau 4) sont du même ordre de grandeur en métropole et dans cette île ${ }^{27}$. Une femme sur six déclare avoir subi des brimades, des critiques répétées et injustes, du dénigrement systématique ou avoir été mise à l'écart. Un peu moins d'une femme sur dix a été insultée, souvent plusieurs fois, et $3 \%$ déplorent une destruction de leur travail ou de leur outil de travail. Enfin deux femmes sur cent se plaignent de harcèlement sexuel. Clients ou usagers, collègues ou supérieurs hiérarchiques, les auteurs des agressions verbales et psychologiques ou de la destruction du travail sont fréquemment des hommes mais aussi des femmes, alors que les agressions sexuelles et physiques sont quasiment toujours perpétrées par des hommes, qui sont le plus souvent des collègues ou des supérieurs.

\footnotetext{
${ }^{26}$ Marylène Lieber. Genre, violences et espaces publics. La vulnérabilité des femmes en question. Paris, Les Presses de Sciences Po, 2008.

${ }^{27}$ Rappelons que les violences au travail ne faisaient pas l'objet d'un module de questions spécifique en Nouvelle-Calédonie et en Polynésie. Les résultats obtenus dans le module « espaces publics » avec la précision «cela s'est passé au travail», mais avec une formulation des questions conçue pour ces espaces publics, ne permettent pas de construire des indicateurs comparables à ceux qui sont présentés pour la métropole et l'île de La Réunion.
} 
Tableau 4 : Proportion (\%) de femmes ayant subi des agressions dans l'exercice de leur profession dans les douze derniers mois, selon le territoire et le type d'agression

\begin{tabular}{|c|c|c|}
\hline Vie professionnelle & $\begin{array}{c}\text { Métropole } \\
N=4756\end{array}$ & $\begin{array}{c}\text { La Réunion } \\
N=615\end{array}$ \\
\hline Agressions verbales & 8,5 & 10,0 \\
\hline Atteintes psychologiques & 16,7 & 16,3 \\
\hline Destructions du travail ou de l'outil de travail & 2,9 & 3,0 \\
\hline Harcèlement sexuel & 2,0 & 2,0 \\
\hline Agressions physiques & 0,6 & 3,0 \\
\hline \multicolumn{3}{|c|}{$\begin{array}{l}\text { Champs : ensemble des femmes ayant exercé un travail rémunéré dans l'année par territoire } \\
\text { Agressions verbales : insultes, menaces de nuire ou de tuer } \\
\text { Atteintes psychologiques: brimades, critiques répétées et injustes, dénigrement } \\
\text { systématique, mise à l'écart } \\
\text { Harcèlement sexuel : attentions gênantes, avances non désirées, exhibition, attouchements, } \\
\text { tentatives de viol ou viol }\end{array}$} \\
\hline
\end{tabular}

Une fois encore, les jeunes femmes sont davantage la cible de toutes ces atteintes, particulièrement des agressions sexuelles, que leurs aînées. Corrélativement, celles qui n'ont pas de conjoint et celles qui ne vivent pas avec lui sont les plus visées. Par ailleurs, quel que soit leur âge, les femmes qui ont vécu des difficultés dans leur enfance ou dans leur adolescence, ayant plus souvent que les autres écourté leur scolarité et se trouvant ainsi en situation d'emploi précaire, connaissent des taux plus élevés d'agressions verbales et d'atteintes psychologiques. Cependant, indépendamment de la qualité de vie pendant la jeunesse, les femmes exerçant des métiers plus qualifiés (cadres et professions intellectuelles, professions intermédiaires) affichent des taux égaux ou plus élevés que les employées et les ouvrières pour tous les types d'atteintes. En fait, plus que la catégorie professionnelle, ce sont des conditions de travail particulières (travail de nuit, agents de conduite, de surveillance ou de l'ordre public...) qui entraînent un accroissement des risques pour les salariées, alors qu'un statut indépendant les réduit, sauf pour les femmes exerçant des professions libérales traditionnellement très masculines - qui sont plus exposées aux atteintes sexuelles.

Les cumuls de violences au travail et dans la vie conjugale sont significatifs : des situations, pas toujours graves lorsqu'elles se produisent séparément, peuvent s'aggraver réciproquement par le biais du stress, des 
manifestations de mal-être ou de fragilité qui exacerbent l'agressivité dans l'autre espace de vie, conjugal ou professionnel ${ }^{28}$.

Près de huit femmes victimes de violences au travail sur dix en ont déjà parlé puisque les incidents se sont très souvent produits en présence de collègues; les agressions physiques sont davantage dénoncées, et très rapidement, alors que les agressions sexuelles sont les plus cachées ou révélées tardivement.

\section{B. - Les violences antérieures}

Dans tous les territoires, on a interrogé les femmes sur les agressions physiques qu'elles avaient pu subir, avant les douze derniers mois, depuis le début de l'âge adulte (on a exclu l'enfance et l'adolescence afin de ne pas tenir compte des châtiments corporels dits éducatifs qui restent beaucoup plus fréquents dans certaines communautés ethniques et culturelles que dans d'autres). Les taux de réponses positives sont trois fois plus élevés en Polynésie et en Nouvelle-Calédonie, où près d'une femme sur deux a enduré de tels sévices, qu'en métropole ou à La Réunion (tableau 5).

Tableau 5 : Proportion (\%) de femmes ayant subi des agressions physiques ou sexuelles dans la vie (douze derniers mois exclus), selon le territoire

\begin{tabular}{|c|c|c|c|c|}
\hline Vie entière & $\begin{array}{c}\text { Métropole } \\
N=6970\end{array}$ & $\begin{array}{c}\text { La Réunion } \\
N=1213\end{array}$ & $\begin{array}{c}\text { Polynésie } \\
N=1001\end{array}$ & $\begin{array}{c}\text { Nlle-Calédonie } \\
N=1012\end{array}$ \\
\hline Agressions physiques* & 15,0 & 14,0 & 42,0 & 44,0 \\
\hline Agressions sexuelles** & 11,0 & 8,2 & 14,0 & 21,5 \\
\hline \multicolumn{5}{|c|}{$\begin{array}{l}\text { Champs : ensemble des femmes enquêtées dans chaque territoire } \\
\text { *: à partir de } 18 \text { ans en métropole et à La Réunion, } 17 \text { ans en Polynésie, } 15 \text { ans en Nouvelle-Calédonie } \\
\text { **: à partir de la naissance } \\
\text { Agressions physiques : gifles, coups, séquestration ou mise à la porte, menaces avec une arme, tentative } \\
\text { de meurtre } \\
\text { Agressions sexuelles : attouchements, tentatives de viol, viols }\end{array}$} \\
\hline
\end{tabular}

Les brutalités physiques et séquestrations ou mises à la porte se sont répétées pour plus d'un tiers des victimes alors que les menaces avec arme et tentatives de meurtre ont rarement été réitérées. Les auteurs des agressions sont d'abord des conjoints ou partenaires au moment des faits - qui demeurent parfois le partenaire actuel - voire des anciens conjoints, puis des hommes et des femmes de la famille. Les proches et les relations de travail sont peu incriminés, alors que les inconnus agissant dans les espaces publics

${ }^{28}$ Elizabeth Brown, Dominique Fougeyrollas-Schwebel \& Maryse Jaspard. « Les paroxysmes de la conciliation: violence au travail et violence du conjoint », Travail, genre et sociétés, $\mathrm{n}^{\circ} 8$, novembre 2002, p. 149-165. 
sont davantage cités en métropole que dans les îles où les femmes sortent rarement seules, le soir en particulier.

L'âge des répondantes n'influence pas sensiblement les taux de violences physiques déclarées : effet de remémoration et seuil de tolérance plus faible parmi les plus jeunes se conjuguent probablement pour contrer une logique d'accroissement des faits avec la durée d'exposition. En revanche, les femmes divorcées, séparées ou même veuves et celles qui ont eu de nombreux partenaires sexuels dans leur vie présentent des taux de victimation plus élevés, que les auteurs soient des partenaires ou anciens partenaires mais aussi des hommes de la parenté, ce qui suggère la survivance d'un contrôle très abusif de la respectabilité des femmes par leur famille. Par ailleurs, les femmes qui ont vécu plusieurs difficultés dans leur jeunesse ont aussi été plus agressées physiquement à l'âge adulte, ce qui traduit une fois de plus la précarité sociale, économique et affective induite par un début de vie troublé.

Les quatre enquêtes exploraient les violences sexuelles éventuellement subies depuis le plus jeune âge jusqu'à l'année précédant l'interrogation. Si l'on s'attendait à une sous-déclaration de faits pénibles, souvent considérés comme humiliants, voire honteux, le taux global de $11 \%$ obtenu dans l'enquête en métropole (tableau 5) était supérieur à ceux des travaux similaires menés à l'époque ${ }^{29}$ et cette estimation, certes minimale, a peut-être participé à une certaine libération de la parole constatée par la suite $^{30}$. Il n'en demeure pas moins que, deux ans plus tard, le taux de 8,2\% enregistré à La Réunion semble confirmer la force du tabou, alors que les fréquences plus élevées constatées en Polynésie et surtout en NouvelleCalédonie seraient plus proches de la réalité.

Lorsqu'elles ne sont pas perpétrées par un conjoint ou un partenaire, ces violences sexuelles touchent la plupart du temps des très jeunes filles, voire des fillettes : plus du tiers de toutes les agressions sexuelles déclarées se sont produites avant les quinze ans de la victime, quel que soit le territoire. Les viols sont alors moins nombreux que les tentatives de viol et les attouchements (tableau 6), mais pour tous ces sévices, les îles du Pacifique distancent de loin la métropole et l'île de La Réunion. Les auteurs sont majoritairement des hommes de la famille (dont le père ou le beau-père, mais aussi des frères, des cousins, des oncles...) ou d'autres hommes connus de la victime.

\footnotetext{
${ }^{29}$ Enquête ACSF 1993, Baromètre Santé 2000, dont les questions étaient moins précises que celles de l'Enveff.

${ }^{30}$ Nathalie Bajos, Michel Bozon et l'équipe CSF. «Les violences sexuelles en France : quand la parole se libère », Population et sociétés, $\mathrm{n}^{\circ} 445$, mai 2008.
} 
Comme pour les violences physiques, on constate une augmentation des taux à l'âge adulte lorsque la personne déclare avoir eu de nombreux partenaires, et un accroissement des taux avant ou après quinze ans lorsque la jeunesse a été marquée par plusieurs difficultés.

Tableau 6 : Proportion (\%) de femmes ayant subi des agressions sexuelles avant l'âge de quinze ans, selon le territoire et le type d'agression

\begin{tabular}{|l|c|c|c|c|}
\hline $\begin{array}{c}\text { Agressions sexuelles } \\
\text { avant 15 ans (au moins } \\
\text { une agression) }\end{array}$ & $\begin{array}{c}\text { Métropole } \\
N=6970\end{array}$ & $\begin{array}{c}\text { La Réunion } \\
N=1213\end{array}$ & $\begin{array}{c}\text { Polynésie } \\
N=1001\end{array}$ & $\begin{array}{c}\text { Nlle-Calédonie } \\
N=1012\end{array}$ \\
\hline Attouchements & 3,3 & 2,0 & 5,1 & 11,6 \\
\hline Tentatives de viol & 0,9 & 0,8 & 1,7 & 2,6 \\
\hline Viol & 0,5 & 0,7 & 1,3 & \\
\hline \multicolumn{2}{|l}{ Champs : ensemble des femmes enquêtées dans chaque territoire } \\
\end{tabular}

\section{CONCLUSION}

Bien que les quatre enquêtes menées dans les années 2000-2002 aient interrogé des personnes vivant dans des contextes sociaux et culturels assez différents, on peut dégager quelques traits communs aux circonstances de la survenue des violences et à la personnalité des victimes :

- Quand on s'intéresse aux situations récentes, le jeune âge est toujours synonyme de surexposition aux risques, traduisant des situations de fragilité, une confrontation aux attirances sexuelles mal contrôlées, mais aussi une moindre tolérance des générations cadettes aux agressions de toutes sortes ;

- L'effet de la formation, du niveau économique et de la profession est finalement peu sensible devant la précarité du statut ou l'éviction du marché du travail, sauf pour des emplois très spécifiques ;

- Les stigmates d'une enfance ou d'une adolescence difficile sont très forts pendant toute la vie et dans tous les espaces ;

- La multiplicité des relations amoureuses ou sexuelles peut être la conséquence d'expériences antérieures malheureuses et entraîner de facto l'accroissement des risques, mais elle attire aussi un jugement négatif des proches qui se permettent des réactions brutales ;

- Par honte, par manque de confiance envers les institutions susceptibles de les aider ou par crainte d'aggraver leur situation, les femmes avaient peu ou n'avaient pas parlé auparavant des 
violences les plus graves qu'elles ont déclarées lors de l'enquête, particulièrement pour ce qui concerne les violences subies dans leurs relations intimes. 


\section{RÉSUMÉ}

\section{Elizabeth Brown. Les enquêtes " Enveff » sur les violences envers les femmes dans la France hexagonale et ultramarine}

Une enquête nationale sur les violences faites aux femmes en France (dite Enveff) a été effectuée dans l'hexagone en 2000 par téléphone auprès d'un échantillon d'environ 7000 femmes. Cette enquête de victimation a porté sur les violences subies par les femmes dans différentes sphères (espaces publics, travail-études, famille et proches, couple, relations avec un ancien conjoint) et différentes périodes de la vie (douze derniers mois, reste de la vie adulte, enfance et adolescence). Elle a également été effectuée à La Réunion (par téléphone), en Nouvelle-Calédonie et en Polynésie française (en face-à-face). Dans tous les territoires, la relation conjugale ou amoureuse, suivie par les espaces publics, est le principal thêâtre des violences déclarées pour les douze derniers mois ; mais les taux de violences déclarées sont nettement plus élevés en Polynésie et en Nouvelle-Calédonie que dans l'hexagone ou à La Réunion. La jeunesse des femmes, la multiplicité de leurs relations amoureuses ou sexuelles et les stigmates d'une enfance ou d'une adolescence difficile sont les facteurs statistiquement les plus associés aux violences déclarées.

\section{ABSTRACT \\ Elizabeth Brown. The "Enveff" studies on violence against women in France and its overseas territories}

In 2000, a national telephonic survey on violence against women (known as Enveff) was undertaken in metropolitan France among a sample of 7,000 women. This survey focused on the violence they endured in various spheres (in public spaces, at work, with family and loved ones, in their current and former relationships) and in different periods of their lives (in the last twelve months, throughout adulthood, and before they were 18 years old). The study was also conducted in Réunion (by telephone), in New Caledonia and in French Polynesia (through face-to-face interviews). In all jurisdictions, the primary sites of violence reported upon within the last twelve months were in romantic relationships, followed by public spaces, and the rates of reported violence were significantly higher in Polynesia and New Caledonia than in metropolitan France and Réunion. The factors most closely associated with reported violence were the youth of the women involved, their having multiple romantic or sexual relationships, and their reporting having suffered stigma related to a difficult childhood or adolescence. 